\title{
Local well-posedness of generalized BBM equations with generalized damping on 1D torus
}

Junjun Kang ${ }^{1}$, Yantao Guo ${ }^{2}$ and Yanbin Tang ${ }^{1 *}$

\section{"Correspondence:}

tangyb@hust.edu.cn

'School of Mathematics and

Statistics, Huazhong University of

Science and Technology, Wuhan,

Hubei 430074, P.R. China

Full list of author information is

available at the end of the article

\begin{abstract}
We consider the periodic initial value problem associated to the generalized Benjamin-Bona-Mahony equation with generalized damping on the one dimensional torus. In contrast to the classical BBM equation, the main difference is that the generalized equation contains two nonlocal operators, and the main difficulty comes from two nonlocal operators. By the fixed point theorem, we prove that the periodic initial value problem is locally well-posed. We also prove that if the solution exists globally in time, it exhibits some asymptotic behavior.
\end{abstract}

MSC: 35A07; 35Q53

Keywords: generalized Benjamin-Bona-Mahony equation; nonlocal operator; generalized damping; local well-posedness

\section{Introduction}

The classical Benjamin-Bona-Mahony (BBM) equation

$$
u_{t}-u_{x x t}+u_{x}+u u_{x}=0
$$

was proposed in [1] as a model for propagation of long waves which incorporates nonlinear dispersive and dissipative effects. It has extensively been studied in the recent literature; see for example [1-10] on the existence and uniqueness of solutions and [11-17] on the global attractors and references therein.

In this paper, we consider the periodic initial value problem of generalized BBM equations with generalized damping on the $1 \mathrm{D}$ torus $\mathbb{T}=\mathbb{R} / 2 \pi \mathbb{Z}$ :

$$
\left\{\begin{array}{l}
u_{t}+L_{p} u_{t}+u_{x}+u u_{x}+M_{\alpha} u=0, \quad x \in \mathbb{T}, t \in(0, T) \\
u(0, x)=u_{0}(x), \quad x \in \mathbb{T}, \\
\int_{\mathbb{T}} u(t, x) d x=0
\end{array}\right.
$$

where the two nonlocal operators are defined by

$$
\widehat{L_{p} u}(k)=|k|^{p+1} \widehat{u}(k), \quad p>0 ;
$$

(c) 2015 Kang et al. This article is distributed under the terms of the Creative Commons Attribution 4.0 International License (http://creativecommons.org/licenses/by/4.0/), which permits unrestricted use, distribution, and reproduction in any medium, provided you give appropriate credit to the original author(s) and the source, provide a link to the Creative Commons license, and indicate if changes were made. 


$$
\widehat{M_{\alpha} u}(k)=|k|^{2 \alpha} \widehat{u}(k), \quad \alpha \in\left(0, \frac{p+1}{2}\right]
$$

and $\widehat{u}(k)$ is the $k$ th Fourier coefficient of $u(t, x)$ in $x$.

For $\alpha=1$, the generalized damping becomes a parabolic damping,

$$
M_{1} u=-u_{x x} \quad \text { as } \widehat{M_{1} u}(k)=|k|^{2} \widehat{u}(k) .
$$

For $\alpha=0$, it is a weak damping,

$$
M_{0} u=u \quad \text { as } \widehat{M_{0}} u(k)=\widehat{u}(k) .
$$

For example, Wang [18] considered the damped BBM equation $u_{t}-u_{t x x}+\gamma\left(u-u_{x x}\right)+$ $u u_{x}=f(x)$ (Introduction, p.134) and the BBM equation with different damping coefficients $u_{t}-u_{t x x}+\gamma u-v u_{x x}+u u_{x}=f(x)$ (Remark 3.2, p.142).

In fact, one can consider more general damping terms. For example, Chehab et al. [19] studied the long-time behavior of the solution of a damped BBM equation

$$
u_{t}-u_{x x t}+u_{x}+u u_{x}+M_{\alpha} u=0, \quad x \in \mathbb{T}[0, L], t \in(0, T)
$$

with

$$
\widehat{M_{\alpha}} u(k)=\gamma_{k} \widehat{u}(k),
$$

and $\left(\gamma_{k}\right)_{k \in \mathbb{Z}}$ are positive real numbers.

In the absence of fractional damping $M_{\alpha} u$, Carvajal and Panthee [20] proved that the Cauchy problem

$$
\left\{\begin{array}{l}
u_{t}+L_{p} u_{t}+u_{x}+\left(u^{k+1}\right)_{x}=0, \quad x \in \mathbb{R}, t \in(0, T), k \in \mathbb{Z}^{+}, \\
u(0, x)=u_{0}(x), \quad x \in \mathbb{R}
\end{array}\right.
$$

is ill-posed for data with lower order Sobolev regularity and in a certain range of the Sobolev regularity, even if the solution exists globally in time, it fails to be smooth.

In this paper we study the generalized BBM equations with the fractional damping terms. In contrast to the classical BBM equation, the main difference is that equation (1.2) contains two nonlocal operators, its dissipation is weaker than the classical BBM equation. In the study of the periodic initial value problem (1.2), the main difficulty is that $L_{p}$ and $M_{\alpha}$ are nonlocal operators. By the fixed point theorem and the Fourier analysis method, similar to [21-23], we prove the local well-posedness of the solution to the problem (1.2).

\section{Local well-posedness}

We define a space

$$
\dot{H}^{\beta}(\mathbb{T})=\left\{u \in L^{2}(\mathbb{T}): \int_{\mathbb{T}} u d x=0, \sum_{k \in \mathbb{Z} \backslash\{0\}}|k|^{2 \beta}|\widehat{u}(k)|^{2}<+\infty\right\}
$$

with the norm

$$
|u|_{\beta}^{2}=\sum_{k \in \mathbb{Z} \backslash\{0\}}|k|^{2 \beta}|\widehat{u}(k)|^{2} .
$$


Then we can obtain the local existence and uniqueness of the solution to the periodic initial value problem (1.2).

Theorem 2.1 Assume $\alpha \in\left(\frac{1}{2}, \frac{p+1}{2}\right]$. If $u_{0}(x) \in \dot{H}^{\alpha}(\mathbb{T})$, there exist a constant $T=C_{0}\left(\left|u_{0}\right|_{\alpha}\right)>$ 0 and a unique solution $u(t, x) \in C\left([0, T], \dot{H}^{\alpha}(\mathbb{T})\right)$. Moreover, for any constant $M>0$, $\left|u_{0}\right|_{\alpha} \leq M,\left|v_{0}\right|_{\alpha} \leq M$, there exists a constant $C_{1}>0$ such that the solutions $u(t, x), v(t, x)$ of the periodic initial value problem (1.2) with the initial data $u_{0}(x) \in \dot{H}^{\alpha}(\mathbb{T})$ and $v_{0}(x) \in$ $\dot{H}^{\alpha}(\mathbb{T})$, respectively, satisfy

$$
|u(t)-v(t)|_{\alpha} \leq C_{1}\left|u_{0}-v_{0}\right|_{\alpha}, \quad \forall t \leq \frac{1}{C_{0} M} .
$$

Proof We first write equation (1.2) in the following form:

$$
\begin{aligned}
u_{t} & =-\left(I+L_{p}\right)^{-1} \partial_{x} u-\left(I+L_{p}\right)^{-1} \partial_{x}\left(\frac{u^{2}}{2}\right)-\left(I+L_{p}\right)^{-1} M_{\alpha} u \\
& =-i \varphi\left(D_{x}\right) u-i \varphi\left(D_{x}\right)\left(\frac{u^{2}}{2}\right)-\varphi_{\alpha}\left(D_{x}\right) u \\
& =-\left[i \varphi\left(D_{x}\right)+\varphi_{\alpha}\left(D_{x}\right)\right] u-i \varphi\left(D_{x}\right)\left(\frac{u^{2}}{2}\right),
\end{aligned}
$$

where

$$
\begin{aligned}
& \widehat{\varphi\left(D_{x}\right)} u(k)=\frac{k}{1+|k|^{p+1}} \widehat{u}(k), \\
& \widehat{\varphi_{\alpha}\left(D_{x}\right)} u(k)=\frac{|k|^{2 \alpha}}{1+|k|^{p+1}} \widehat{u}(k), \quad k \in \mathbb{Z} .
\end{aligned}
$$

Then we get

$$
u(t)=S_{t} u_{0}-i \int_{0}^{t} S_{t-\tau} \varphi\left(D_{x}\right)\left(\frac{u^{2}}{2}\right) d \tau,
$$

where

$$
S_{t} u_{0}=\sum_{k \in \mathbb{Z} \backslash\{0\}} e^{i k x} e^{-\frac{i k+|k|^{2 \alpha}}{1+|k| p+1} t} \widehat{u_{0}}(k) .
$$

We define a map

$$
\Phi(u(t))=S_{t} u_{0}-i \int_{0}^{t} S_{t-\tau} \varphi\left(D_{x}\right)\left(\frac{u^{2}}{2}\right) d \tau
$$

and a closed ball

$$
\bar{B}(\mathbb{T})=\left\{u(t, x) \in C\left([0, T], \dot{H}^{\alpha}(\mathbb{T})\right):\left|u(t)-u_{0}\right|_{\alpha} \leq 3\left|u_{0}\right|_{\alpha}\right\}
$$

We now prove that $\Phi$ has a unique fixed point in $\bar{B}(\mathbb{T})$.

Step one: $\Phi$ is onto, that is, for $u(t) \in \bar{B}(\mathbb{T})$ we have $\Phi(u(t)) \in \bar{B}(\mathbb{T})$. 
According to the definition of the norm $|u(t)|_{\alpha}$, we get

$$
\begin{aligned}
\left|S_{t} u_{0}\right|_{\alpha}^{2} & =\sum_{k \in \mathbb{Z} \backslash\{0\}}|k|^{2 \alpha}\left|\widehat{S_{t} u_{0}}(k)\right|^{2} \\
& =\sum_{k \in \mathbb{Z} \backslash\{0\}}|k|^{2 \alpha}\left|e^{-\frac{i k+|k|^{2 \alpha}}{1+|k|^{p+1}}} \widehat{u_{0}}(k)\right|^{2} \\
& =\sum_{k \in \mathbb{Z} \backslash\{0\}}|k|^{2 \alpha} e^{-\frac{2|k|^{2 \alpha}}{1+|k|^{+1}} t}\left|\widehat{u_{0}}(k)\right|^{2} \\
& \leq \sum_{k \in \mathbb{Z} \backslash\{0\}}|k|^{2 \alpha}\left|\widehat{u_{0}}(k)\right|^{2}=\left|u_{0}\right|_{\alpha}^{2}
\end{aligned}
$$

and

$$
\begin{aligned}
\left|S_{t-\tau} \varphi\left(D_{x}\right)\left(\frac{u^{2}}{2}\right)\right|_{\alpha}^{2} & =\sum_{k \in \mathbb{Z} \backslash\{0\}}|k|^{2 \alpha} \mid S_{t-\tau} \widehat{\left.\varphi\left(D_{x}\right)\left(u^{2} / 2\right)(k)\right|^{2}} \\
& =\sum_{k \in \mathbb{Z} \backslash\{0\}}|k|^{2 \alpha}\left|e^{-\frac{i k+|k|^{2 \alpha}\left(t+||^{p+1}\right.}{1+\tau)}} \frac{k}{1+|k|^{p+1}} \widehat{\left(u^{2} / 2\right)}(k)\right|^{2} \\
& \leq \sum_{k \in \mathbb{Z} \backslash\{0\}}|k|^{2 \alpha}\left|\widehat{\left(u^{2} / 2\right)}(k)\right|^{2} \\
& =\frac{1}{4} \sum_{k \in \mathbb{Z} \backslash\{0\}}|k|^{2 \alpha}\left|\widehat{u^{2}}(k)\right|^{2} \\
& =\frac{1}{4}\left|u^{2}\right|_{\alpha}^{2} \leq C|u|_{\alpha}^{4},
\end{aligned}
$$

the last inequality comes from the fact that $\dot{H}^{\alpha}(\mathbb{T})$ is an algebra for $\alpha>\frac{1}{2}$.

Putting the above two inequalities into (2.6) we have

$$
\begin{aligned}
|\Phi(u)|_{\alpha} & \leq\left|S_{t} u_{0}\right|_{\alpha}+\int_{0}^{t}\left|S_{t-\tau} \varphi\left(D_{x}\right)\left(\frac{u^{2}}{2}\right)\right|_{\alpha} d \tau \\
& \leq\left|u_{0}\right|_{\alpha}+C \int_{0}^{t}|u(\tau)|_{\alpha}^{2} d \tau \\
& \leq\left|u_{0}\right|_{\alpha}+C T \sup _{t \in[0, T]}|u(t)|_{\alpha}^{2} .
\end{aligned}
$$

Since $u(t) \in \bar{B}(\mathbb{T})$ and

$$
|u(t)|_{\alpha}-\left|u_{0}\right|_{\alpha} \leq\left|u(t)-u_{0}\right|_{\alpha} \leq 3\left|u_{0}\right|_{\alpha}
$$

we have $|u(t)|_{\alpha} \leq 4\left|u_{0}\right|_{\alpha}$ and

$$
\begin{aligned}
\left|\Phi(u(t))-u_{0}\right|_{\alpha} & \leq|\Phi(u(t))|_{\alpha}+\left|u_{0}\right|_{\alpha} \leq 2\left|u_{0}\right|_{\alpha}+16 C T\left|u_{0}\right|_{\alpha}^{2} \\
& \leq 3\left|u_{0}\right|_{\alpha}, \quad \text { if } 0<T<\frac{1}{16 C\left|u_{0}\right|_{\alpha}} .
\end{aligned}
$$

Therefore, for $T \in\left(0, \frac{1}{16 C\left|u_{0}\right|_{\alpha}}\right)$, we have $\Phi(u(t)) \in \bar{B}(\mathbb{T})$ for $u(t) \in \bar{B}(\mathbb{T})$. 
Step two: $\Phi$ is a contractive mapping on $\bar{B}(\mathbb{T})$.

Let $u(t), v(t) \in \bar{B}(\mathbb{T})$. Since

$$
\begin{aligned}
|\Phi(u(t))-\Phi(v(t))|_{\alpha}^{2} & =\left|\int_{0}^{t} S_{t-\tau} \varphi\left(D_{x}\right)\left(\frac{u^{2}}{2}-\frac{v^{2}}{2}\right) d \tau\right|_{\alpha}^{2} \\
& \leq \int_{0}^{t} \sum_{k \in \mathbb{Z} \backslash\{0\}}|k|^{2 \alpha}\left|e^{-\frac{i k+\left.k\right|^{2 \alpha}}{1+|k|+1}(t-\tau)} \frac{k}{1+|k|^{p+1}}\left(\frac{u^{2}}{2}-\frac{v^{2}}{2}\right)(k)\right|^{2} d \tau \\
& \left.\leq C \int_{0}^{t} \sum_{k \in \mathbb{Z} \backslash\{0\}}|k|^{2 \alpha} \mid \widehat{\left(u^{2}-v^{2}\right.}\right)\left.(k)\right|^{2} d \tau \\
& =C \int_{0}^{t}\left|u^{2}-v^{2}\right|_{\alpha}^{2} d \tau \\
& \leq 2 C \int_{0}^{t}\left(|u|_{\alpha}^{2}+|v|_{\alpha}^{2}\right)|u-v|_{\alpha}^{2} d \tau,
\end{aligned}
$$

due to $u(t), v(t) \in \bar{B}(\mathbb{T})$, we have

$$
|\Phi(u(t))-\Phi(v(t))|_{\alpha}^{2} \leq 32 C\left(\left|u_{0}\right|_{\alpha}^{2}+\left|v_{0}\right|_{\alpha}^{2}\right) T \sup _{t \in[0, T]}|u(t)-v(t)|_{\alpha}^{2},
$$

that is,

$$
\sup _{t \in[0, T]}|\Phi(u(t))-\Phi(v(t))|_{\alpha} \leq 4 \sqrt{2 C T\left(\left|u_{0}\right|_{\alpha}^{2}+\left|v_{0}\right|_{\alpha}^{2}\right)} \sup _{t \in[0, T]}|u(t)-v(t)|_{\alpha} .
$$

Therefore, $\Phi$ is a contractive mapping on $\bar{B}(\mathbb{T})$ if $4 \sqrt{2 C T\left(\left|u_{0}\right|_{\alpha}^{2}+\left|v_{0}\right|_{\alpha}^{2}\right)}<1$, that is,

$$
0<T<\frac{1}{32 C\left(\left|u_{0}\right|_{\alpha}^{2}+\left|v_{0}\right|_{\alpha}^{2}\right)} .
$$

Thanks to the Banach fixed point theorem, $\Phi$ has a unique fixed point $u(t)$ such that $u(t)=$ $\Phi(u(t))$, that is, there exists a unique solution of the periodic initial value problem (1.2).

Step three: the continuity of solution with the initial data.

Let $u(t)$ and $v(t)$ be solutions of the periodic initial value problem (1.2) with the initial data $u_{0}$ and $v_{0}$, respectively, such that $\left|u_{0}\right|_{\alpha} \leq M,\left|v_{0}\right|_{\alpha} \leq M$. For $t \in[0, T]$, the Duhamel principle gives us the following formula:

$$
u(t)-v(t)=S_{t}\left(u_{0}-v_{0}\right)-i \int_{0}^{t} S_{t-\tau} \varphi\left(D_{x}\right)\left(\frac{u^{2}}{2}-\frac{v^{2}}{2}\right) d \tau
$$

hence

$$
\begin{aligned}
|u(t)-v(t)|_{\alpha} & \leq\left|S_{t}\left(u_{0}-v_{0}\right)\right|_{\alpha}+\int_{0}^{t}\left|S_{t-\tau} \varphi\left(D_{x}\right)\left(\frac{u^{2}}{2}-\frac{v^{2}}{2}\right)\right|_{\alpha} d \tau \\
& \leq\left|u_{0}-v_{0}\right|_{\alpha}+C_{0}\left(|u(t)|_{\alpha}+|v(t)|_{\alpha}\right) T \sup _{t \in[0, T]}|u(t)-v(t)|_{\alpha} \\
& \leq\left|u_{0}-v_{0}\right|_{\alpha}+4 C_{0}\left(\left|u_{0}\right|_{\alpha}+\left|v_{0}\right|_{\alpha}\right) T \sup _{t \in[0, T]}|u(t)-v(t)|_{\alpha} \\
& \leq\left|u_{0}-v_{0}\right|_{\alpha}+8 C_{0} M T \sup _{t \in[0, T]}|u(t)-v(t)|_{\alpha}
\end{aligned}
$$


if $T<\frac{1}{8 C_{0} M}$, there exists a constant $C_{1}=\frac{1}{1-8 C_{0} M T}>0$ such that

$$
|u(t)-v(t)|_{\alpha} \leq \sup _{t \in[0, T]}|u(t)-v(t)|_{\alpha} \leq C_{1}\left|u_{0}-v_{0}\right|_{\alpha} .
$$

The proof is complete.

\section{Asymptotic behavior of the solution}

We first consider the corresponding problem with the linear equation

$$
\left\{\begin{array}{l}
u_{t}+L_{p} u_{t}+u_{x}+M_{\alpha} u=0, \quad x \in \mathbb{T}, t \in(0, T) \\
u(0, x)=u_{0}(x), \quad x \in \mathbb{T}, \\
\int_{\mathbb{T}} u(t, x) d x=0
\end{array}\right.
$$

If $u(t) \in L^{2}(\mathbb{T}), \forall t>0$, then the $k$ th Fourier coefficient $\widehat{u}_{k}(t)$ of $u(t, x)$ in $x$ satisfies

$$
\left(1+|k|^{p+1}\right) \widehat{u}_{k}^{\prime}(t)+\left(i k+|k|^{2 \alpha}\right) \widehat{u}_{k}(t)=0, \quad k \in \mathbb{Z}
$$

that is,

$$
\widehat{u}_{k}(t)=e^{-\frac{i k+|k|^{2 \alpha}}{1+|k| p^{+1}} \widehat{u}_{k}(0)}
$$

Therefore, we have

$$
|u|_{\frac{p+1}{2}}^{2}=\sum_{k \in \mathbb{Z} \backslash\{0\}}|k|^{p+1}\left|\widehat{u}_{k}(t)\right|^{2}=\sum_{k \in \mathbb{Z} \backslash\{0\}}|k|^{p+1} e^{-\frac{2|k|^{2 \alpha}}{1+|k|^{p+1} t}\left|\widehat{u}_{k}(0)\right|^{2} .}
$$

Theorem 3.1 If $u_{0}(x) \in \dot{H}^{\frac{p+1}{2}}(\mathbb{T})$, then the unique solution $u(t, x)$ of the periodic initial value problem (3.1) satisfies

$$
|u(t)|_{\frac{p+1}{2}}^{2} \leq\left|u_{0}\right|_{\frac{p+1}{2}}^{2}, \quad 0<\alpha \leq \frac{p+1}{2} .
$$

Furthermore, we have

$$
\begin{aligned}
|u(t)|_{\alpha}^{2} \leq \frac{1}{e t}\left|u_{0}\right|_{\frac{p+1}{2}}^{2}, \quad 0<\alpha<\frac{p+1}{2}, \forall t>0, \\
|u(t)|_{\alpha}^{2} \leq e^{-t}\left|u_{0}\right|_{\frac{p+1}{2}}^{2}, \quad \alpha=\frac{p+1}{2}, \forall t>0 .
\end{aligned}
$$

Proof Equation (3.2) implies that

$$
\begin{aligned}
|u|_{\frac{p+1}{2}}^{2} & =\sum_{k \in \mathbb{Z} \backslash\{0\}}|k|^{p+1} e^{-\frac{2|k|^{2 \alpha}}{1+|k|^{p+1}}\left|\widehat{u}_{k}(0)\right|^{2}} \\
& \leq \sum_{k \in \mathbb{Z} \backslash\{0\}}|k|^{p+1}\left|\widehat{u}_{k}(0)\right|^{2} \\
& =\left|u_{0}\right|_{\frac{p+1}{2}}^{2}, \quad 0<\alpha \leq \frac{p+1}{2} .
\end{aligned}
$$


On the other hand, for $0<\alpha<\frac{p+1}{2}$ we have

$$
\begin{aligned}
|u|_{\alpha}^{2} & =\sum_{k \in \mathbb{Z} \backslash\{0\}}|k|^{2 \alpha} e^{-\frac{2|k|^{2 \alpha}}{1+|k|^{p+1}}\left|\widehat{u}_{k}(0)\right|^{2}} \\
& =\sum_{k \in \mathbb{Z} \backslash\{0\}}|k|^{p+1} \frac{1+|k|^{p+1}}{|k|^{p+1}} \frac{|k|^{2 \alpha}}{1+|k|^{p+1}} e^{-\frac{2|k|^{2 \alpha}}{1+|k|^{p+1}}\left|\widehat{u}_{k}(0)\right|^{2}} \\
& \leq \sum_{k \in \mathbb{Z} \backslash\{0\}}|k|^{p+1} \varphi_{\alpha}(k) e^{-\varphi_{\alpha}(k) t}\left|\widehat{u}_{k}(0)\right|^{2},
\end{aligned}
$$

where

$$
\varphi_{\alpha}(k)=\frac{2|k|^{2 \alpha}}{1+|k|^{p+1}}, \quad 0<\alpha<\frac{p+1}{2}, k \in \mathbb{Z} \backslash\{0\} .
$$

Since the function $x e^{-x t}$ is uniformly bounded by $\frac{1}{e t}$, we have

$$
|u|_{\alpha}^{2} \leq \frac{1}{e t} \sum_{k \in \mathbb{Z} \backslash\{0\}}|k|^{p+1}\left|\widehat{u}_{k}(0)\right|^{2}=\frac{1}{e t}\left|u_{0}\right|_{\frac{p+1}{2}}^{2} .
$$

For $\alpha=\frac{p+1}{2}$, we have

$$
\begin{aligned}
|u|_{\frac{p+1}{2}}^{2} & =\sum_{k \in \mathbb{Z} \backslash\{0\}}|k|^{p+1} e^{-\frac{\left.2|k|\right|^{p+1}}{1+|k|^{p+1} t}\left|\widehat{u}_{k}(0)\right|^{2}} \\
& \leq \sum_{k \in \mathbb{Z} \backslash\{0\}}|k|^{p+1} e^{-t}\left|\widehat{u}_{k}(0)\right|^{2} \\
& =e^{-t} \sum_{k \in \mathbb{Z} \backslash\{0\}}|k|^{p+1}\left|\widehat{u}_{k}(0)\right|^{2} \\
& =e^{-t}\left|u_{0}\right|_{\frac{p+1}{2}}^{2} .
\end{aligned}
$$

The proof is complete.

We now deal with the nonlinear equation (1.2), that is, $u_{t}+L_{p} u_{t}+u_{x}+u u_{x}+M_{\alpha} u=0$. We can find similar kind of decreasing properties but less explicit than in the linear case.

Theorem 3.2 If $u_{0}(x) \in \dot{H}^{\frac{p+1}{2}}(\mathbb{T})$, then the unique solution $u(t, x)$ of the periodic initial value problem (1.2) satisfies

$$
\lim _{t \rightarrow+\infty}|u(t)|_{\frac{p+1}{2}}^{2}=0
$$

Proof Since $\int_{\mathbb{T}} u u_{x} d x=0, \int_{\mathbb{T}} u^{2} u_{x} d x=0$, and

$$
\begin{aligned}
\int_{\mathbb{T}} u\left(u_{t}+L_{p} u_{t}\right) d x & =\pi \frac{d}{d t}\left(\sum_{k \in \mathbb{Z} \backslash\{0\}}\left|\widehat{u}_{k}(t)\right|^{2}+\sum_{k \in \mathbb{Z} \backslash\{0\}}|k|^{p+1}\left|\widehat{u}_{k}(t)\right|^{2}\right) \\
& =\pi \frac{d}{d t} \sum_{k \in \mathbb{Z} \backslash\{0\}}\left(1+|k|^{p+1}\right)\left|\widehat{u}_{k}(t)\right|^{2},
\end{aligned}
$$




$$
\int_{\mathbb{T}} u M_{\alpha} u d x=2 \pi \sum_{k \in \mathbb{Z} \backslash\{0\}}|k|^{2 \alpha}\left|\widehat{u}_{k}(t)\right|^{2}=2 \pi|u(t)|_{\alpha}^{2}
$$

The equation and zero mean condition in (1.2) imply that

$$
\frac{1}{2} \frac{d}{d t} \sum_{k \in \mathbb{Z} \backslash\{0\}}\left(1+|k|^{p+1}\right)\left|\widehat{u}_{k}(t)\right|^{2}+|u(t)|_{\alpha}^{2}=0,
$$

hence

$$
\frac{d}{d t} \sum_{k \in \mathbb{Z} \backslash\{0\}}\left(1+|k|^{p+1}\right)\left|\widehat{u}_{k}(t)\right|^{2}=-2|u(t)|_{\alpha}^{2} \leq 0 .
$$

It implies that $\sum_{k \in \mathbb{Z} \backslash\{0\}}\left(1+|k|^{p+1}\right)\left|\widehat{u}_{k}(t)\right|^{2}$ is decreasing in $t$, so we have

$$
\sum_{k \in \mathbb{Z} \backslash\{0\}}\left(1+|k|^{p+1}\right)\left|\widehat{u}_{k}(t)\right|^{2} \leq \sum_{k \in \mathbb{Z} \backslash\{0\}}\left(1+|k|^{p+1}\right)\left|\widehat{u}_{k}(0)\right|^{2}, \quad \forall t \geq 0 .
$$

Therefore,

$$
\begin{aligned}
|u(t)|_{\frac{p+1}{2}}^{2} & =\sum_{k \in \mathbb{Z} \backslash\{0\}}|k|^{p+1}\left|\widehat{u}_{k}(t)\right|^{2} \\
& \leq \sum_{k \in \mathbb{Z} \backslash\{0\}}\left(1+|k|^{p+1}\right)\left|\widehat{u}_{k}(0)\right|^{2} \\
& \leq 2\left|u_{0}\right|_{\frac{p+1}{2}}^{2}, \quad \forall t \geq 0 .
\end{aligned}
$$

Equation (3.15) and $u_{0}(x) \in \dot{H}^{\frac{p+1}{2}}$ lead to $u(t) \in \dot{H}^{\frac{p+1}{2}}$ and then $u(t) \in \dot{H}^{\alpha}$ for $\frac{1}{2}<\alpha \leq \frac{p+1}{2}$.

On the other hand, (3.13) implies that $\sum_{k \in \mathbb{Z} \backslash\{0\}}\left(1+|k|^{p+1}\right)\left|\widehat{u}_{k}(t)\right|^{2}$ is decreasing in $t$ and bounded below by zero, then the limit

$$
\lim _{t \rightarrow+\infty} \sum_{k \in \mathbb{Z} \backslash\{0\}}\left(1+|k|^{p+1}\right)\left|\widehat{u}_{k}(t)\right|^{2}
$$

exists, we denote it by $A$.

Denote

$$
\lim _{t \rightarrow+\infty}|u(t)|_{\alpha}^{2}=B
$$

If $B>0$, for large enough $t$, we have $|u(t)|_{\alpha}^{2}>B / 2$, then there is a constant $T>0$ such that

$$
\frac{d}{d t} \sum_{k \in \mathbb{Z} \backslash\{0\}}\left(1+|k|^{p+1}\right)\left|\widehat{u}_{k}(t)\right|^{2}<-B, \quad t>T
$$

hence

$$
\sum_{k \in \mathbb{Z} \backslash\{0\}}\left(1+|k|^{p+1}\right)\left|\widehat{u}_{k}(t)\right|^{2}<\sum_{k \in \mathbb{Z} \backslash\{0\}}\left(1+|k|^{p+1}\right)\left|\widehat{u}_{k}(T)\right|^{2}-B(t-T),
$$


and then

$$
\lim _{t \rightarrow+\infty} \sum_{k \in \mathbb{Z} \backslash\{0\}}\left(1+|k|^{p+1}\right)\left|\widehat{u}_{k}(t)\right|^{2} \leq-\infty .
$$

This contradiction leads to $\lim _{t \rightarrow+\infty}|u(t)|_{\alpha}^{2}=B=0$, that is,

$$
\lim _{t \rightarrow+\infty} \sum_{k \in \mathbb{Z} \backslash\{0\}}|k|^{2 \alpha}\left|\widehat{u}_{k}(t)\right|^{2}=0
$$

then $\forall k \in \mathbb{Z}, \lim _{t \rightarrow+\infty}\left|\widehat{u}_{k}(t)\right|^{2}=0$, therefore we have

$$
\lim _{t \rightarrow+\infty} \sum_{k \in \mathbb{Z} \backslash\{0\}}\left(1+|k|^{p+1}\right)\left|\widehat{u}_{k}(t)\right|^{2}=A=0
$$

and

$$
\lim _{t \rightarrow+\infty} \sum_{k \in \mathbb{Z} \backslash\{0\}}|k|^{p+1}\left|\widehat{u}_{k}(t)\right|^{2}=0, \quad \text { i.e. } \lim _{t \rightarrow+\infty}|u(t)|_{\frac{p+1}{2}}^{2}=0 .
$$

The proof is complete.

Remark 3.1 This paper gives the local well-posedness for the subcritical index $\alpha>\frac{1}{2}$. The interesting case would be to consider the supercritical case $0<\alpha<\frac{1}{2}$ and the critical case $\alpha=\frac{1}{2}$. In the supercritical case $0<\alpha<\frac{1}{2}$, there will be less dissipation, so the dispersive part comes to play a principal role. In the cases $0<\alpha<\frac{1}{2}$ and $\alpha=\frac{1}{2}, \dot{H}^{\alpha}(\mathbb{T})$ is not an algebra, we must find another way to establish the estimates on the nonlinear term. We will consider the supercritical case in future work.

\section{Competing interests}

The authors declare that they have no competing interests.

\section{Authors' contributions}

JK carried out the nonlocal operator, YG carried out the Benjamin-Bona-Mahony equation, and YT carried out the well-posedness. All authors read and approved the final manuscript.

\section{Author details}

'School of Mathematics and Statistics, Huazhong University of Science and Technology, Wuhan, Hubei 430074, P.R. China.

${ }^{2}$ School of Mathematics and Statistics, Xuchang University, Xuchang, Henan 461000, P.R. China.

\section{Acknowledgements}

The authors are grateful to the anonymous referees for helpful comments and suggestions that greatly improved the presentation of this paper. This work was supported by National Natural Science Foundation of China (Grant Nos. 11471129 and 11272277$)$

Received: 24 September 2015 Accepted: 20 November 2015 Published online: 03 December 2015

\section{References}

1. Benjamin, TB, Bona, JL, Mahony, JJ: Model equations for long waves in nonlinear dispersive systems. Philos. Trans. R. Soc. Lond. 272, 47-78 (1972)

2. Avrin, J: The generalized Benjamin-Bona-Mahony equation in $R^{n}$ with singular initial data. Nonlinear Anal. 11, 139-147 (1987)

3. Avrin, J, Goldstein, JA: Global existence for the Benjamin-Bona-Mahony equation in arbitrary dimensions. Nonlinear Anal. 9, 861-865 (1985)

4. Bona, JL, Bryant, PJ: A mathematical model for long waves generated by wavemakers in nonlinear dispersive systems Proc. Camb. Philos. Soc. 73, 391-405 (1973)

5. Bona, JL, Dougalis, VA: An initial and boundary value problem for a model equation for propagation of long waves. J. Math. Anal. Appl. 75, 503-522 (1980) 
6. Chen, YM: Remark on the global existence for the generalized Benjamin-Bona-Mahony equations in arbitrary dimension. Appl. Anal. 30, 1-15 (1988)

7. Goldstein, JA, Wichnoski, BJ: On the Benjamin-Bona-Mahony equation in higher dimensions. Nonlinear Anal. 4, 665-675 (1980)

8. Guo, YT, Wang, M, Tang, YB: Higher regularity of global attractor for a damped Benjamin-Bona-Mahony equation on R. Appl. Anal. 94(9), 1766-1783 (2015)

9. Medeiros, LA, Miranda, MM: Weak solutions for a nonlinear dispersive equation. J. Math. Anal. Appl. 59, 432-441 (1977)

10. Medeiros, LA, Menzala, GP: Existence and uniqueness for periodic solutions of the Benjamin-Bona-Mahony equation. SIAM J. Math. Anal. 8, 792-799 (1977)

11. Astaburuaga, MA, Bisognin, E, Bisognin, V, Fernandez, C: Global attractors and finite dimensionality for a class of dissipative equations of BBM's type. Electron. J. Differ. Equ. 1998, 25 (1998)

12. Celebi, AO, Kalantarov, VK, Polat, M: Attractors for the generalized Benjamin-Bona-Mahony equation. J. Differ. Equ. 157, 439-451 (1999)

13. Chueshov, I, Polat, M, Siegmund, S: Gevrey regularity of global attractor for generalized Benjamin-Bona-Mahony equation. Mat. Fiz. Anal. Geom. 11, 226-242 (2004)

14. Stanislavova, M, Stefanov, A, Wang, B: Asymptotic smoothing and attractors for the generalized Benjamin-Bona-Mahony equation on $R^{3}$. J. Differ. Equ. 219, 451-483 (2005)

15. Wang, B: Regularity of attractors for the Benjamin-Bona-Mahony equation. J. Phys. A 31, 7635-7645 (1998)

16. Wang, B, Fussner, DW, Bi, C: Existence of global attractors for the Benjamin-Bona-Mahony equation in unbounded domains. J. Phys. A 40, 10491-10504 (2007)

17. Wang, B, Yang, W: Finite dimensional behaviour for the Benjamin-Bona-Mahony equation. J. Phys. A 30, 4877-4885 (1997)

18. Wang, M: Long time dynamics for a damped Benjamin-Bona-Mahony equation in low regularity. Nonlinear Anal. TMA 105, 134-144 (2014)

19. Chehab, JP, Garnier, P, Mammeri, Y: Long-time behavior of solutions of a BBM equation with generalized damping (2014) arXiv:1402.5009

20. Carvajal, X, Panthee, M: On ill-posedness for the generalized Benjamin-Bona-Mahony equation. Discrete Contin. Dyn. Syst. 34(11), 4565-4576 (2014)

21. Wang, $M$, Tang, YB: Attractors in $H^{2}$ and $L^{2 p-2}$ for reaction diffusion equations on unbounded domains. Commun. Pure Appl. Anal. 12(2), 1111-1121 (2013)

22. Wang, M, Tang, YB: On dimension of the global attractor for 2D quasi-geostrophic equations. Nonlinear Anal., Real World Appl. 14, 1887-1895 (2013)

23. Wang, $M$, Tang, YB: Long time dynamics of $2 D$ quasi-geostrophic equations with damping in $L^{p}$. J. Math. Anal. Appl. $412,866-877(2014)$

\section{Submit your manuscript to a SpringerOpen ${ }^{\ominus}$ journal and benefit from:}

- Convenient online submission

- Rigorous peer review

- Immediate publication on acceptance

- Open access: articles freely available online

- High visibility within the field

- Retaining the copyright to your article 